\title{
Reply to "Pain Control After Bariatric Surgery: We Still Need More Answers"
}

\author{
Jun Jie $\mathrm{Ng}^{1}$ (D) $\cdot$ Asim Shabbir ${ }^{1}$
}

Published online: 29 March 2018

(C) Springer Science+Business Media, LLC, part of Springer Nature 2018

\section{Dear Editor,}

We would like to thank Dr. Mark C Kendall for his letter and interest in our manuscript "A Multimodal Analgesic Protocol Reduces Opioid-Related Adverse Events and Improves Patient Outcomes in Laparoscopic Sleeve Gastrectomy" [1]. We would like to clarify the methodological issues that have been brought up by Dr. Mark C Kendall.

Firstly, all patients who underwent laparoscopic sleeve gastrectomy in this study did not receive any form or neuraxial or regional anaesthesia. Routine wound infiltration with bupivacaine $0.5 \%$ was performed after skin closure for all patients. There was a significant reduction in the use of diclofenac during the postoperative period in patients who were managed within MAP $(55 \pm 74.1 \mathrm{mg}$ vs. $0.7 \pm 6.1 \mathrm{mg}$, $p<0.001$ ), although this was not reported in our original study. Some authors have suggested that non-steroidal antiinflammatory drugs (NSAIDs) should be discontinued in the immediate postoperative phase after bariatric surgery due to an increased risk of bleeding [2]. The reduction in the use of diclofenac in patients who were treated with the MAP is expected as NSAIDs were not included within the protocol. Secondly, we agree that a paucity of data regarding intraoperative remifentanil usage and duration of surgery could confound our data, although we do not expect the duration of surgery to be significantly different between both groups.

Despite the limitations of our study, we feel that the benefits of a MAP are clear, and a MAP should be adopted in institutions offering bariatric surgery.

\section{References}

1. Ng JJ, Leong WQ, Tan CS, et al. A multimodal analgesic protocol reduces opioid-related adverse events and improves patient outcomes in laparoscopic sleeve gastrectomy. Obes Surg. 2017;27: 3075-81.

2. Elrazek AEMAA, Elbanna AEM, Bilasy SE. Medical management of patients after bariatric surgery: principles and guidelines. World Journal of Gastrointestinal Surgery. 2014;6:220-8.
Jun Jie $\mathrm{Ng}$

jun_jie_ng@nuhs.edu.sg

Asim Shabbir

cfsasim@nus.edu.sg

1 Division of Upper Gastrointestinal Surgery, Department of Surgery, National University Health System, Singapore 119228, Singapore 